\title{
High precision reconstruction of electromagnetic showers in the nuclear emulsions of the OPERA experiment
}

\author{
Fulvio Laudisio ${ }^{1 \dagger}$ \\ CISAS \& INFN Padova \\ Via Marzolo 8, Padova (Italy) \\ E-mail: fulvio.laudisioepd.infn.it
}

We present an automatic procedure developed to reconstruct electromagnetic EM showers in the nuclear emulsions of the OPERA neutrino experiment. It is an entirely new procedure based on emulsion images acquired with $1 \mu \mathrm{m}$ pitch tomography. The enhanced $3 \mathrm{D}$ accuracy is used in the reconstruction of particles' tracks and for the evaluation of shower energies in interesting neutrino event topologies detected by OPERA.

The European Physical Society Conference on High Energy Physics

5-12 July, 2017

Venice

\footnotetext{
${ }^{1}$ Speaker

${ }^{\dagger}$ on behalf of the Opera Collaboration 


\section{Introduction}

We present an automatic procedure developed to reconstruct EM showers in the nuclear emulsions of the OPERA neutrino experiment. OPERA was designed to investigate muon neutrino oscillations using so called Emulsion Cloud Chambers (ECC) [1]. Lead plates constituting the target mass were interleaved with nuclear emulsion films providing high spatial resolution. Each target unit was composed by $561 \mathrm{~mm}$ thick lead plates and 57 emulsion films. Each emulsion film had two sensitive layers poured on the two sides of a plastic film. The full target was composed by 150.000 ECC arranged in planes interleaved with scintillating fibers. A muon spectrometer completed the target.

The reconstruction and measurement of EM showers is complicated by the noise due to aging of emulsion films and background tracks. Usually, electromagnetic showers are reconstructed by connecting tracks between adjacent films; the procedure is also complemented by visual inspections.

The presented technique, developed to reduce the need of human intervention, can automatically detect and reconstruct electromagnetic showers. Moreover it has been possible to establish a correlation between the reconstructed topology and the shower energy. Image acquisition is performed by a CCD camera mounted on a motorized stage, images are acquired with a $1 \mu \mathrm{m}$ pitch in depth. Silver grains are identified and used to reconstruct particles' tracks. By this precise 3D tomographic technique it is possible to achieve a spatial resolution of $0.1 \mu \mathrm{m}$ and an angular resolution of $1 \mathrm{mrad}$. Such accuracies allowed to reduce combinatorial correlations while keeping track reconstruction efficiency close to $100 \%$ and purity close to $95 \%$. Primary electron/photon energy can be correlated to the number of reconstructed tracks in emulsion. The calibration has been optimized using a sample of Monte Carlo events.

Identification and reconstruction efficiencies of electromagnetic showers are discussed in the following. The procedure is used to study short lived particle candidate events in ECC, possibly associated with tau and charm production.

\section{Image analysis and track reconstruction}

The dedicated image analysis procedure was based on the European Emulsion Scanning System (ESS) [2] employed in the OPERA experiment. The ESS consists of an optical microscope equipped with a computer-controlled motorized stage, a dedicated optical system and a CMOS camera for fast image acquisition. Several images are grabbed at equally spaced depth levels $(Z)$ in each field of view and processed using a Matrox Odyssey frame grabber/vision processor. After processing the silver grains appear as black dots in the image, called clusters. The ESS track pattern recognition algorithm is based on these clusters to which are assigned planar coordinates $(\mathrm{XY})$; the third coordinate $(\mathrm{Z})$ is the vertical position of the scanning stage.

The spatial resolution has been improved by refining the vertical coordinate of each grain. Image grabbing was performed with vertical pitch of $1 \mu \mathrm{m}, 40$ images are grabbed per each emulsion sensitive layer ( $42 \mu m$ thick) instead of the 16 images used in the ESS. Taking advantage of this enriched sampling, the $\mathrm{Z}$ coordinate could be refined furthermore interpolating shadow effects between consecutive images. Using three consecutive images, the 3D positions of grains are obtained by fitting the local gray level minima (Fig.1).

A 3D track along one emulsion film is reconstructed when a row of aligned grains is found, in any direction, in the field of view. The tracking algorithm searches for aligned sequences of grains around a fixed direction; the angular acceptance can be defined in a wide range (typically $25 \mathrm{mrad}$ for specific track search and $500 \mathrm{mrad}$ for general scan purposes). To reduce the time needed, the scanned volume is divided into adjacent slices aligned with the chosen direction; the transverse (XY) dimension of the volume is set by the maximum allowed tolerance, initially set to $1 \mu \mathrm{m}$. A sequence of three aligned grains in a volume is the trigger signal for track building, aligned grains are searched for in the selected volume and a linear fit 
procedure is applied.

Taking advantage of the sub-micron spatial resolution of nuclear emulsions, stringent requirements on the grain displacement from the fitted trajectory can be applied on both longitudinal and transverse coordinates. The fitting residuals are $0.15 \mu \mathrm{m}$ so the transverse tolerance is eventually reduced to $0.45 \mu \mathrm{m}$. Spurious background grains can be efficiently rejected using this method; this procedure allows automatic reconstruction of electron-positron tracks.

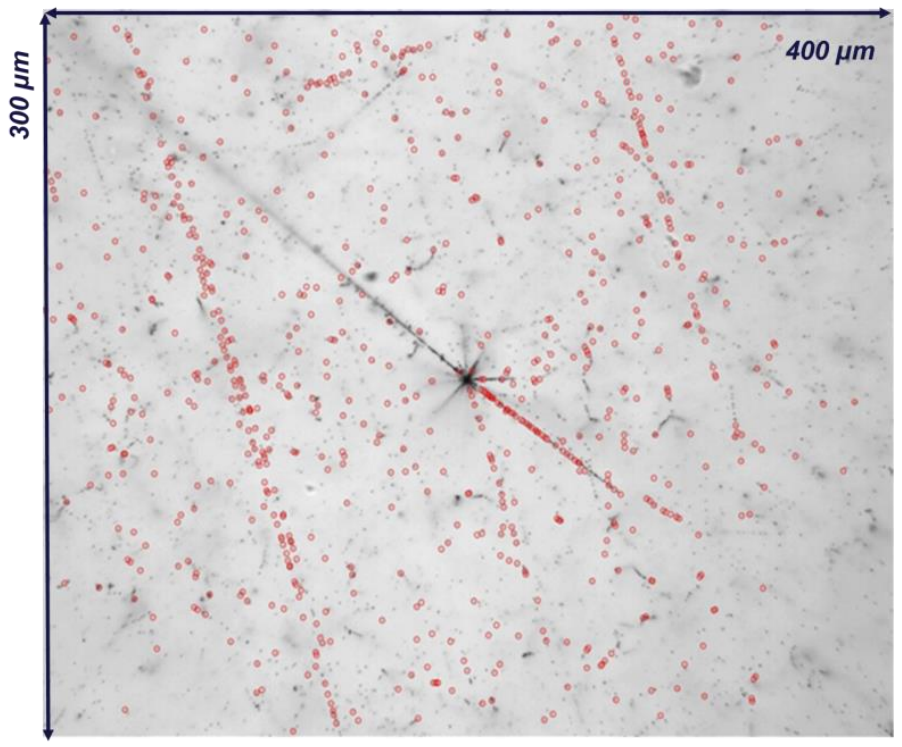

Figure 1: Grabbed image of exposed nuclear emulsion after development on a single field of view. Selected grains are highlighted by red circles.

\section{Electromagnetic shower reconstruction}

The EM shower energy is associated to the track multiplicity at interaction vertex by the Heitler model. Each electron (positron) emits a bremsstrahlung photon in one radiation length $\left(X_{0}\right)$; each photon converts into an electron-positron pair in the same length. The depth $X_{\max }$ where tracks multiplicity is maximum is defined as:

$$
\begin{gathered}
X_{0}=\frac{\left(716.4 \mathrm{~g} . \mathrm{cm}^{-2}\right) A}{Z(Z+1) \ln \left(\frac{287}{\sqrt{Z}}\right)} \\
X_{\max }=\frac{X_{0} \ln \frac{E_{0}}{E_{c}}}{\ln 2}
\end{gathered}
$$

$E_{c}$ is the critical energy in emulsion-lead cells. The track multiplicity is measured at $X_{\max }$ is used to evaluate the energy $E_{0}$ of the primary particle as follows:

$$
N\left(X_{\max }\right)=\frac{E_{0}}{E_{c}}
$$

The track multiplicity of an EM shower is evaluated by determining sets of linked tracks along the scanned volume. The volume is spanned by an iterative process which collects all the tracks sharing at least one link. The link between two tracks is established setting the threshold of two 
quantities, the angle between the two tracks and the distance between the two intersections of the tracks with the intermediate plane lying between them.

Threshold values for linking parameters were defined by means of MonteCarlo simulation over a set of $v_{\mathrm{e}}$ events. Three sets of thresholds (Small, Medium, Large) were applied and compared (see Table 1). A correlation between shower energy and tracks multiplicity is obtained ad shown in Fig. 2. The achieved energy resolution is suitable for OPERA events kinematical study (Fig. $3)$.

\begin{tabular}{||c||c|c||}
\hline & Angle $(\mathbf{r a d})$ & Distance $(\boldsymbol{\mu m})$ \\
\hline \hline Small & 0.040 & 40 \\
\hline \hline Medium & 0.050 & 50 \\
\hline \hline Large & 0.060 & 60 \\
\hline
\end{tabular}

Table 1: Set of parameters used to perform track linking in an emulsion volume

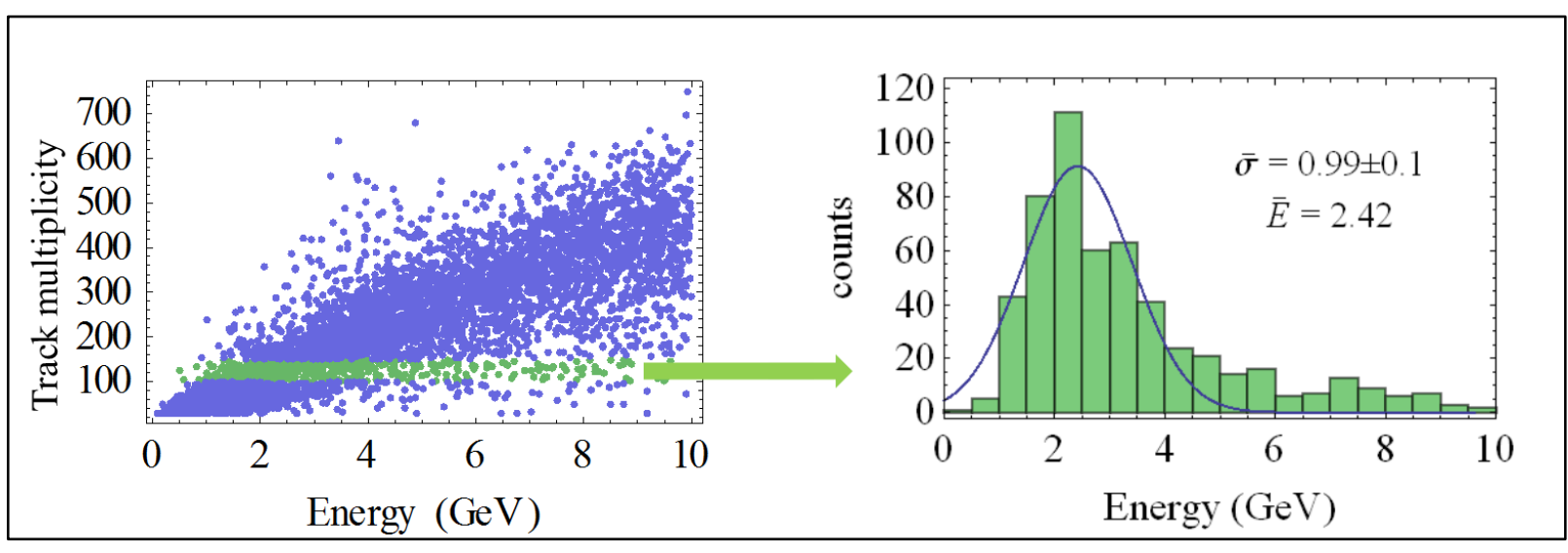

Figure 2: Left plot: Track multiplicity versus neutrino energy; the distribution is obtained using a sample of 30 emulsion films and the set of thresholds referred as Large. The samples having a track multiplicity between 100 and 150 are highlighted in green. Right plot: Energy estimation for the selected sample.

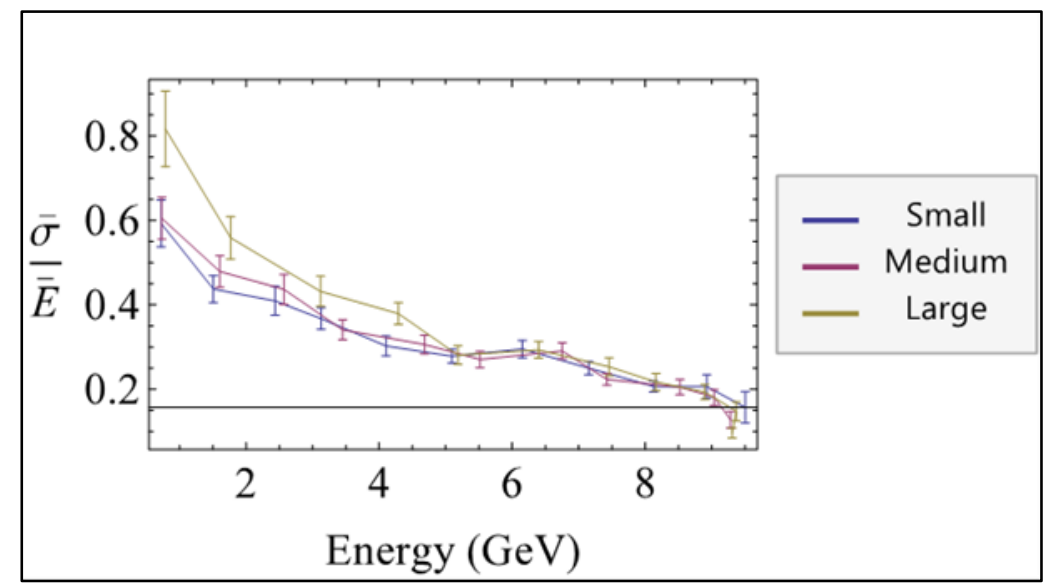

Figure 3: Energy resolution versus neutrino energy. The resolution is worse at lower energies because of the induced cosmic ray background and Compton electrons. 


\section{References}

[1] N. Agafonova et al, OPERA collaboration, Discovery of $\tau$ Neutrino Appearance in the CNGS Neutrino Beam with the OPERA Experiment, PRL 115 (2015) 121802

[2] N. Armenise et al, High-speed particle tracking in nuclear emulsion by last-generation automatic microscopes, Nucl.Instrum.Meth.A 551 (2005) 261-270 